\title{
Hepatic Microcirculation in the Perfused Cirrhotic Rat Liver
}

France Varin and P.-Michel Huet

The Clinical Research Center and Department of Medicine of the Hôpital Saint-Luc and the Université de Montréal, Montreal, Quebec H2X $3 J 4$ Canada

\section{Abstract}

Liver microcirculation in the perfused rat liver was assessed by the multiple indicator dilution technique. Comparative studies were carried out in noncirrhotic rats and in rats with cirrhosis secondary to chronic exposure to phenobarbital and carbon tetrachloride. The alterations of the sinusoidal bed were characterized by changes in the displacement of hepatic venous outflow curves of various diffusible substances (labeled albumin, sucrose, and water) relative to that of labeled erythrocytes (vascular reference). Outflow recoveries of lidocaine (a substance that penetrates the liver cell membrane freely and completely) and of labeled microspheres (15 $\mu \mathrm{m}$ diam) were also appraised. In all cirrhotic rats, unimodal erythrocytes and albumin curves were obtained. The sinusoidal space was significantly decreased when compared with normal rats $(P<0.001)$ and the total space accessible to albumin became progressively restricted. In seven cirrhotic rats, the profiles of labeled sucrose and water curves were compatible with a flow-limited diffusion and the total distribution volumes were not significantly different from values found in noncirrhotic rats $(P=N S)$, which indicates that sucrose and water were still able to diffuse into an extravascular space not accessible to albumin. In the other cirrhotic rats, labeled sucrose and water curves showed progressive bimodal changes not compatible with a flow-limited diffusion. Such alterations were not due to large intrahepatic shunts, since only $0.25 \%$ of the $15-\mu \mathrm{m}$ microspheres were recovered in the outflow of cirrhotic rats. However, an early lidocaine outflow peak related in time to the peak erythrocyte curve was observed in cirrhotic, but not in noncirrhotic, rats. Lidocaine recovery varied greatly in cirrhotic rats and appeared to increase as the liver disease progressed. These data can be explained by capillarization of sinusoids and/ or by the development of channels with poor permeability. Electron microscopic observations of these rat livers favored the latter. Thus, in cirrhotic rat liver, two kinds of alteration are likely: (a) the vascular space is decreased with collagenization of the extravascular space, limiting the diffusion of large molecules such as albumin; and (b) small channels with poorly permeable walls develop, limiting the diffusion of small molecules such as lidocaine, sucrose, and water. Large intrahepatic shunts are not a common feature.

This work was presented at the 34th Annual Meeting of the American Association for the Study of Liver Diseases, Chicago, IL 1983, and appeared in abstract form in Hepatology. 1983. 3:826.

Dr. Varin is a Fellow of the Medical Research Council of Canada, and Dr. Huet is a Chercheur-Boursier of the Fonds de la Recherche en Santé du Québec. Address reprint requests to Dr. Huet.

Received for publication 19 December 1984 and in revised form 24 June 1985

J. Clin. Invest.

(c) The American Society for Clinical Investigation, Inc.

0021-9738/85/11/1904/09 \$1.00

Volume 76, November 1985, 1904-1912

\section{Introduction}

In addition to the reduction of liver cell mass, alterations in the liver microcirculation contribute to the progressive limitation of blood-liver exchange and to the reduced elimination of endogenous and exogenous substances. Collagenization of the space of Disse with the formation of a basement membrane (1) and intrahepatic, portohepatic shunts $(2,3)$ are the two major anatomical alterations described in long-standing human cirrhosis (4). Using the multiple indicator dilution technique, Goresky's (5) approach, we previously reported (6) that capillarization and collagenization of hepatic sinusoids were of particular importance in patients with alcoholic liver cirrhosis. However, in that study, the presence of intrahepatic shunts was not directly appraised.

In order to evaluate the relative importance of these two alterations in hepatic microcirculation, capillarization, and shunts, we carried out studies using the multiple indicator dilution technique in the isolated perfused liver of cirrhotic rats. Chronic administration of carbon tetrachloride $\left(\mathrm{CCl}_{4}\right)$ to phenobarbital-treated rats was chosen as the experimental model because it has been shown (7) to yield morphologic alterations resembling human cirrhosis.

In this model, we defined the hepatic venous outflow patterns resulting from portal vein injection of labeled erythrocytes $(\mathrm{RBCs})^{1}$ and plasma-dissolved labeled substances not metabolized by the liver (albumin, sucrose, and water). In normal liver, RBCs are confined to the vascular space (vascular reference), while plasma-dissolved substances gain access to the extravascular space (the space of Disse and hepatocytes) through fenestrae in and between the endothelial cells: albumin $(69,000 \mathrm{~mol} \mathrm{wt})$ and sucrose (342 mol wt) diffuse in extracellular spaces that are inversely related to their molecular weights, and water diffuses in the cellular space (5). This produces a major delay in, and a decrease in the magnitude of, the labeled albumin, sucrose, and water curves with respect to that of labeled RBCs, which can be analyzed using the flow-limited linear two-compartment model described by Goresky (5). This method yields estimates of sinusoidal blood volume and of the extravascular volume of distribution of diffusible substances (peak-time method). Extravascular volumes can also be calculated in a more direct manner from the transit times (transit-time method) (8), and will always be correct as long as all of the label is recovered at the outflow, even in a system with reduced permeability.

In cirrhotic liver, if conditions for a flow-limited distribution are no longer fulfilled, the extravascular volume measured by the peak-time method may depart widely from the volume measured by the transit-time method. This disparity can result either from shunting, in which both reference and diffusible labels pass through the vasculature with little difference in their transit times, or from collagenization and capillarization, in which the devel-

1. Abbreviation used in this paper: $\mathrm{RBC}(\mathrm{s})$, erythrocyte. 
opment of a new barrier limits diffusion in the extravascular space.

In order to differentiate between these two conditions, we modified Goresky's (5) approach by using: (a) microspheres larger than the diameter of normal sinusoids, which should all be trapped within the sinusoids if no intrahepatic shunts (larger than their diameter) are present, and $(b)$ lidocaine, a lipophilic substance, which is completely removed from plasma during a single passage through the normal liver. The latter substance was used in order to evaluate the possible development of small channels with poorly permeable walls.

\section{Methods}

Animals. Micronodular cirrhosis was induced following the procedure previously described by Proctor and Chatamra (7). Briefly, male SpragueDawley rats with a starting weight of $\sim 170 \mathrm{~g}$ were given phenobarbital sodium $(35 \mathrm{mg} / \mathrm{dl})$ in their drinking water; the first dose of $\mathrm{CCl}_{4}$ was given $10 \mathrm{~d}$ later. Under our conditions, the acute nonlethal dose of $\mathrm{CCl}_{4}$, given intragastrically to rats under phenobarbital induction, was found to be $0.08 \mathrm{ml}$, delivered in $1 \mathrm{ml}$ of corn oil. Successive doses of $\mathrm{CCl}_{4}$ were thereafter varied individually in order to maintain a critical level of damage, as reflected by the daily body weight fluctuation of each rat. $\mathrm{CCl}_{4}$ was administered once a week chronobiologically, using a curved animal-feeding needle with a ball-end (16G-3; Lessard et Fils, St-Hubert, Quebec). At the start of the study, halothane anesthesia was used for intubation of the esophagus and administration of intragastric $\mathrm{CCl}_{4}(7)$. However, over the long term, the rats developed a resistance to the halothane and the duration of inhalation needed to be increased to induce anesthesia. We therefore tried intubation without anesthesia and, in our opinion, skilled manipulation of the animals was proven to be sufficient to preclude the rupture of esophageal varices, if present. Halothane anesthesia potentiated the acute injury and accelerated the onset of ascites; however, $\mathrm{CCl}_{4}$ administration without anesthesia was finally preferred because a more progressive insult with less mortality and a more predictive response was observed.

The duration of treatment varied between 8 and $15 \mathrm{wk}$. A minimal delay of $7 \mathrm{~d}$ after the last dose of $\mathrm{CCl}_{4}$ was allowed before microcirculation studies were undertaken. Histological confirmation of cirrhosis was obtained in all cases on biopsy specimen fixed for light and electron microscopic examination. Phenobarbital-treated rats received vehicle without $\mathrm{CCl}_{4}$, while control rats received no treatment. All animals were kept on a standard light cycle and were fed ad lib. with a stock pellet dish (Lab Chows, Ralston Purina Co., St. Louis, MO).

Materials. ${ }^{51} \mathrm{Cr}$-labeled canine RBCs were prepared with $\mathrm{Na}_{2}{ }^{51} \mathrm{CrO}_{2}$ ( $290 \mu \mathrm{Ci} / \mathrm{mg}$; Frosst, Dorval, Quebec) as described by Wagner (9). ${ }^{99} \mathrm{mTc}-$ labeled albumin was obtained by addition of ${ }^{99}$ metastable Tc (produced by a technetium generator in St-Luc Hospital) to human serum albuminstannous chloride complex (Frosst). ${ }^{14} \mathrm{C}$-labeled sucrose $(3.7 \mathrm{mCi} / \mathrm{mmol}$ ) and ${ }^{3} \mathrm{H}$-labeled water $(0.25 \mathrm{mCi} / \mathrm{ml})$ were ordered from New England Nuclear, Boston, MA. ${ }^{57} \mathrm{Co}$-labeled NEN-TRAC TM microspheres (New England Nuclear) having a mean diameter of $15.8 \mu \mathrm{m}$ and a specific activity of $13.7 \mathrm{mCi} / \mathrm{g}$ were used.

Bovine serum albumin, fraction V (Sigma Chemical Co., St. Louis, MO), phenobarbital sodium (BDH Chemical, Toronto, Ontario), and lidocaine hydrochloride (Astra Pharmaceuticals Canada Ltd.) were purchased. $\mathrm{CCl}_{4}$ and all other chemical reagents were certified American Chemical Standard grade (Fisher Scientific Co., Pittsburgh, PA).

Analytical procedures. ${ }^{51} \mathrm{Cr},{ }^{99} \mathrm{mTc}$, and ${ }^{57} \mathrm{Co}$ were quantitated in a Beckman Gamma 8000 counter (Beckman Instruments Inc., Palo Alto, CA). ${ }^{14} \mathrm{C}$ and ${ }^{3} \mathrm{H}$ activities were determined in a Beckman LS-8000 liquid scintillation counter, using Biofluor (New England Nuclear) as the emulsifier scintillation cocktail. Quenching was corrected by an automated external standard method. Manipulation of samples and standards as well as appropriate cross-over corrections for the gamma and beta activities have been described elsewhere (6). Lidocaine plasma concentra- tions in pooled samples were determined by high performance liquid chromatography, using a technique described earlier (10). A calibration curve was obtained for each set of data.

Liver isolation and perfusion. Rats were anesthetized with pentobarbital $(50 \mathrm{mg} / \mathrm{kg}$, i.p.) and the liver was perfused in situ by a method previously described (11) using a perfusion apparatus (MX/Ambec Perfuser two/ten, MX International Inc., Aurora, CO). Before cannulation of the portal vein and inferior vena cava, the in vivo portal venous pressure was measured, by direct puncture of the vessel whenever technically possible, using a 23-gauge needle connected to a hydrostatic manometer. The common bile duct was cannulated using PE-10 tubing and the bile was collected at 10 -min intervals.

The perfusion medium consisted of Krebs-Henseleit buffer, pH 7.44, saturated with a mixture of $95 \% \mathrm{O}_{2}$ and $5 \% \mathrm{CO}_{2}$, which contained $20 \%$ ( vol/vol) prewashed canine RBCs, $1.5 \%$ (wt/vol) bovine serum albumin, and $100 \mathrm{mg} \%$ glucose.

The liver remained in situ and its temperature was maintained at $37^{\circ} \mathrm{C}$. The perfusate was recirculated with a Masterflex pump (ColePalmer Instrument Co., Chicago, IL) through a stainless steel filter. A pressure regulator was used to damp down pulsatile flow. The perfusion rate through the liver was first set at $16 \mathrm{ml} \cdot \mathrm{min}^{-1}$ and was then modified to obtain a portal venous perfusion pressure similar to the portal venous pressure observed at the time of laparotomy. The perfusion rate was measured volumetrically after diversion of the venous outflow return. In each preparation, the $\mathrm{pH}, \mathrm{pO}_{2}$, and $\mathrm{pCO}_{2}$ of perfusate and hepatic venous outflow were determined and adjusted. The viability of the perfused liver was assessed by gross appearance, perfusion pressure, bile production, and oxygen consumption (12).

Multiple indicator dilution procedures. After a 20-min stabilization period, the perfusion circuit was diverted on the effluent side and one bolus was injected in the portal vein. The injection mixture consisted of ${ }^{51} \mathrm{Cr}$-labeled canine RBCs $\left(10^{5} \mathrm{dpm}\right)$ with a hematocrit matched to that of the perfusion medium, ${ }^{99} \mathrm{mTc}$-labeled albumin $\left(1.5 \times 10^{5} \mathrm{dpm}\right),{ }^{14} \mathrm{C}$ labeled sucrose $\left(1.5 \times 10^{6} \mathrm{dpm}\right)$, and ${ }^{3} \mathrm{H}$-labeled water $\left(3 \times 10^{6} \mathrm{dpm}\right)$. In phenobarbital-treated rats and cirrhotic rats, another bolus was simultaneously injected in the portal vein and consisted of $1 \mathrm{mg}$ of lidocaine in the form of a $500-\mathrm{mg} \%$ solution in physiological saline. The exact volume of injection was determined gravimetrically in a preweighed syringe, assuming a density of 1.0 ; this volume averaged 0.1 and $0.2 \mathrm{ml}$ for each respective bolus. The total hepatic venous outflow was collected in serial tubes at the rate of one tube per second for $40 \mathrm{~s}$ and then at 3$\mathrm{s}$ intervals during the last $120 \mathrm{~s}$. Two aliquots from each tube were obtained: one for the determination of gamma and beta activity, processed as previously described (6), and the other for lidocaine determination. Each aliquot was centrifuged and $50 \mu \mathrm{l}$ of the supernatant from four consecutive samples was pooled and kept frozen until assayed for unchanged lidocaine.

2 min later, a second set of dilution curves was obtained. The injection mixture contained ${ }^{51} \mathrm{Cr}$-labeled canine RBCs $\left(10^{5} \mathrm{dpm}\right)$ with the hematocrit matched to that of the perfusate and ${ }^{57} \mathrm{Co}-$ labeled $15-\mu \mathrm{m} \mathrm{mi-}$ crospheres $\left(7 \times 10^{5} \mathrm{dpm}, \sim 7,000\right.$ beads). The microspheres were sonicated immediately before their addition to the injection mixture. The total hepatic venous outflow was collected in serial tubes at $1-\mathrm{s}$ intervals for $40 \mathrm{~s}$.

The outflow activity (dpm per milliliter) or concentration (micrograms per milliliter) was divided by the total amount injected (dpm or micrograms) in order to provide a basis for comparison between the tracers, yielding a pattern expressed in terms of the outflow fraction per milliliter of blood (i.e., a reciprocal volume) (5). Linear extrapolation of the downslope to infinity was carried out on a semilogarithmic plot and the areas under the time outflow fraction per milliliter curves were calculated. The outflow fraction for lidocaine was plotted against the middle time of the collection period. The outflow recovery of each label was calculated as the ratio of its area to that of the simultaneously injected labeled RBCs (5).

Calculations. Data were analyzed according to Goresky's (5) flowlimited distribution model. The mean catheter transit time was subtracted from the mean transit time $(t)$ calculated using the method of Meier and 
Zierler (13). Large-vessel transit time $\left(t_{0}\right)$ was calculated according to the formula $t_{\mathrm{o}}=\left[t_{1 / 2 \mathrm{pRBC}}\left(1+\gamma_{\mathrm{DIF}}\right)-t_{1 / 2 \mathrm{pDIF}}\right] / \gamma_{\mathrm{DIF}}$, where $t_{1 / 2 \mathrm{pRBC}}$ is the transit time at the half-peak of the labeled RBC dilution curve and $t_{1 / 2 \mathrm{pDIF}}$ is the transit time at the half-peak of the labeled DIF dilution curve; $\left(1+\gamma_{\text {DIF }}\right)$ was calculated as the ratio of the peak RBC outflow fraction per milliliter to the peak DIF outflow fraction per milliliter. The sinusoidal volume $\left(V_{\sin }\right)$ was calculated the $\mathrm{RBC}$ curve as: $V_{\sin }\left(\mathrm{ml} \cdot \mathrm{g}^{-1}\right.$ liver $)=\left(t_{\mathrm{RBC}(\mathrm{s})}-t_{\mathrm{\alpha (s)}}\right) \times$ blood flow $\left(\mathrm{ml} \cdot \mathrm{s}^{-1} \cdot \mathrm{g}^{-1}\right.$ liver $)$. For each diffusible label, the extravascular space was calculated: $(a)$ from the peak-time concentrations (peak-time volume) as: $V_{\mathrm{sin}} \times \gamma_{\mathrm{DIF}}\left(\mathrm{ml} \cdot \mathrm{g}^{-1}\right.$ liver) (5); these values were corrected for hematocrit for albumin and sucrose, substances confined to the plasma compartment of blood; and $(b)$ from the delay in transit times (transit-time volume) as: blood flow $\times\left(t_{\mathrm{DIF}}-t_{\mathrm{RBC}}\right)$ $\left(\mathrm{ml} \cdot \mathrm{g}^{-1}\right.$ liver) (8); these values were corrected for hematocrit for albumin and sucrose.

Large intrahepatic shunts (percent) were calculated as (area under the curve $\cdot$ microspheres/AUC RBC) $\times 100$; lidocaine recovery (percent) was calculated as (AUC $\cdot$ lidocaine/AUC $\cdot \mathrm{RBC}) \times 100$.

Ultrastructural examinations. In all cirrhotic rats, the liver was prepared for light and electron microscopy. The liver was perfused with phosphate-buffered $2 \%$ glutaraldehyde, postfixed in osmium tetroxide, dehydrated through alcohol, and embedded in Epon 812. Regenerative areas of parenchymal tissue were randomly selected by phase-contrast light microscopy. Thin sections of these areas were stained with lead citrate and uranyl acetate for electron microscopy and 35-mm pictures were taken with an Hitachi $\mathrm{H} 300$.

\section{Results}

\section{Experimental cirrhosis and perfusion}

The micronodular cirrhosis yield was similar in rats with or without halothane anesthesia and no difference was found in the histological pattern. Therefore, the 5 rats anesthetized using halothane and the 11 others were all analyzed together. In the procedure described by Proctor and Chatamra (7), the individual response to intragastric administration of $\mathrm{CCl}_{4}$ was used to calibrate the subsequent dose given to the same animal. Under our conditions, this method was found to induce micronodular cirrhosis with ascites in $\sim 60 \%$ of the rats after a treatment period averaging $12 \mathrm{wk}$. Mortality averaged $30 \%$ and occurred mostly at the beginning of the study, when halothane anesthesia was used. The remaining $10 \%$ of rats did not respond to treatment.
Transient ascitic spikes were observed in most rats after 6 wk of treatment, but these generally disappeared before the next dose was administered. Livers were hypertrophied in these animals, as indicated in Table I, which summarizes the mean values of the liver weight/body weight ratio. A significant volume of ascites $(>10 \mathrm{ml})$, persisting after cessation of treatment, was observed in only three rats, and was associated with the presence of an atrophic liver.

In noncirrhotic rats, the portal venous pressure was $12.5 \pm 1.6$ $\mathrm{cm} \mathrm{H}_{2} \mathrm{O}(n=10)$, whereas in cirrhotic rats, the portal pressure was $18.1 \pm 2.6 \mathrm{~cm} \mathrm{H}_{2} \mathrm{O}(n=11)(P<0.001)$. During perfusion, both cirrhotic and noncirrhotic livers maintained a normal, uniform color and indicators of hepatic viability remained stable with an adequate oxygen consumption (Table I). Perfusion rates per gram of liver were significantly higher in normal rats than in phenobarbital-treated or cirrhotic rats $(P<0.001)$. Portal venous perfusion pressure was significantly increased in all cirrhotic rats $(P<0.001)$, although perfusion rates were similar to those in phenobarbital-treated rats $(P>0.10)$ or even lower than in normal rats $(P<0.001)$ (Table $I)$. The recovery of various nonmetabolized labels compared with that of labeled RBCs was $0.92 \pm 0.04$ for labeled albumin, $0.97 \pm 0.05$ for labeled sucrose, and $0.95 \pm 0.08$ for labeled water, and was similar in cirrhotic and noncirrhotic rats.

\section{Outflow dilution curves}

Normal and phenobarbital-treated rats. A typical set of hepatic venous outflow curves obtained in a phenobarbital-treated rat liver is illustrated in the left-hand panel of Fig. 1. After an initial delay (catheter transit time), labeled RBCs first appeared in the outflow, reached peak concentration in a few seconds, and then declined monoexponentially with time. The outflow curves for labeled albumin and sucrose are displaced in relation to that of the RBC curve, showing a lower peak concentration and longer transit time. The displacement is much greater for the labeled water outflow curve after its diffusion into the cellular compartment.

In phenobarbital-treated rats (Fig. 1), unchanged lidocaine emerged substantially later than the labeled water curve and its outflow fraction per milliliter rose to a much later and lower peak.

Table I. Parameters of In Situ Perfused Rat Liver (Mean \pm SD)

\begin{tabular}{|c|c|c|c|c|c|}
\hline Rats & Liver weight & $\begin{array}{l}\text { Liver/body } \\
\text { weight ratio }\end{array}$ & Perfusion rate & $\begin{array}{l}\text { Portal venous } \\
\text { perfusion pressure }\end{array}$ & $\mathrm{O}_{2}$ Consumption \\
\hline & $g$ & & $\begin{array}{l}\mathrm{ml} \cdot \min ^{-1} \cdot g \\
-1 \text { liver }\end{array}$ & $\mathrm{cm} \mathrm{H}_{2} \mathrm{O}$ & $\begin{array}{l}\mu \mathrm{mol} \text {. } \\
\min ^{-1} \\
g^{-1} \text { liver }\end{array}$ \\
\hline \multicolumn{6}{|l|}{ Normal } \\
\hline$(n=7)$ & $12.72 \pm 1.56$ & $0.035 \pm 0.002$ & $1.32 \pm 0.24$ & $12.35 \pm 1.68$ & $1.17 \pm 0.36$ \\
\hline \multicolumn{6}{|c|}{ Phenobarbital-treated } \\
\hline$(n=8)$ & $18.43 \pm 2.35$ & $0.047 \pm 0.009$ & $0.84 \pm 0.12$ & $13.61 \pm 1.53$ & $1.57 \pm 0.11$ \\
\hline \multicolumn{6}{|l|}{ Cirrhotic* } \\
\hline \multicolumn{6}{|l|}{ Group A } \\
\hline$(n=7)$ & $20.99 \pm 2.08$ & $0.048 \pm 0.004$ & $0.78 \pm 0.01$ & $17.25 \pm 2.75$ & $1.10 \pm 0.13$ \\
\hline \multicolumn{6}{|l|}{ Group B } \\
\hline$(n=4)$ & $21.30 \pm 1.60$ & $0.050 \pm 0.002$ & $0.78 \pm 0.01$ & $19.75 \pm 1.55$ & $1.04 \pm 0.06$ \\
\hline \multicolumn{6}{|l|}{ Group C } \\
\hline$(n=5)$ & $14.73 \pm 2.85$ & $0.038 \pm 0.007$ & $0.90 \pm 0.02$ & $24.76 \pm 3.58$ & $0.69 \pm 0.05$ \\
\hline
\end{tabular}

* Cirrhotic rats are classified in three groups according to the different patterns of labeled sucrose and water curves as detailed in results. 


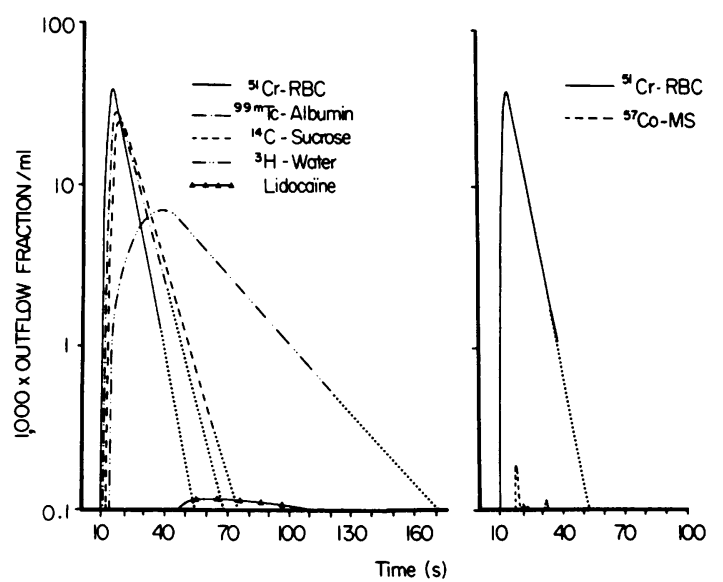

Figure 1. Typical multiple indicator dilution pattern (left) and outflow recovery of microspheres (MS) (right) obtained in an in situ perfused phenobarbital-treated rat liver. The outflow recovery of lidocaine was $0.20 \%$; that of microspheres was $0.10 \%$.

The later injection of a mixture of labeled RBCs and $15-\mu \mathrm{m}$ microspheres in the same liver preparation gave the following outflow profile (Fig. 1, right-hand panel): during the passage of labeled RBCs, some ${ }^{57} \mathrm{Co}$ activity was observed in a few nonconsecutive samples; the overall recovery did not attain the profile of an indicator dilution curve.

The mean values obtained after treatment of the multiple indicator dilution data in seven normal rats are listed in Table II. Average mean transit-time $(t)$ values for the labeled RBCs $(9.00 \mathrm{~s})$, albumin (12.20 s), sucrose (13.81 s), and water (31.98 s) were corrected for the inflow and outflow catheter transit time. The vascular space $\left(V_{\text {sin }}\right)$ represented $0.186 \mathrm{ml} \cdot \mathrm{g}^{-1}$ liver, and the albumin extravascular space (transit-time volume) averaged $0.057 \mathrm{ml} \cdot \mathrm{g}^{-1}$ liver. The sucrose extravascular space was slightly larger than that of albumin $\left(0.086 \mathrm{ml} \cdot \mathrm{g}^{-1}\right)$; the percentage of recovered microspheres varied between 0.00 and 0.24 .

As expected, the liver weight in phenobarbital-treated rats was significantly higher than in normal ones (Table I) and $t$ for different labels was slightly longer (Table II); however, there was no significant difference in $V_{\text {sin }}$ and extravascular volumes, expressed per gram of liver weight $(P>0.10)$. Microsphere recovery was $0.11 \pm 0.10 \%$, but, as in normal rats, the outflow pattern never attained the profile of a dilution curve. Lidocaine recovery was $0.4 \pm 0.5 \%$, being higher than $1.0 \%$ in only one case $(1.50 \%)$.

Cirrhotic rats. Results are presented in Tables I and II. Typical outflow patterns are shown in Figs. 2-4. In all cirrhotic rats, unimodal labeled RBC and albumin curves, with smooth monothetically decreasing down-slopes, were obtained that were compatible with a flow-limited distribution. However, the hepatic venous outflow curves for labeled sucrose and water displayed three different patterns and rats were separated accordingly into three groups. In seven rats, both labeled sucrose and water curves were unimodal, with the kind of profile that would be expected for substances undergoing flow-limited distribution in their accessible extravascular space (Fig. 2) (group A). In four rats, labeled sucrose curves exhibited a different and distinctive form: in each case, the up-slope was slightly delayed in relation to labeled RBC and albumin curves, and the down-slope exhibited a biexponential character. Labeled water curves in these rats were still unimodal (Fig. 3) (group B). Finally, in five cirrhotic rats, both labeled sucrose and water curves were bimodal (Fig. 4) (group C).

Labeled $R B C$ and albumin studies. In cirrhotic rats, $V_{\text {sin }}$ was significantly reduced when compared with normal $(P<0.001)$ and phenobarbital-treated rats $(P<0.005)$. The extravascular volumes accessible to albumin were found to cover a wide range from near normal to markedly decreased values. The total volume accessible to labeled albumin ( $V_{\text {sin }}$ plus albumin extravascular space) was significantly reduced in the 16 cirrhotic rats when compared with those that were noncirrhotic $(P<0.001)$ (Fig. 5).

According to the flow-limited model, the albumin extravascular volume calculated using the peak-time concentration (peaktime volume) should be closely related to the one calculated using transit time (transit-time volume). Fig. 6 illustrates the variation of transit-time volume with the peak-time volume for albumin in the 16 cirrhotic rats: a significant correlation was found between both estimates $(r=0.916, P<0.001)$ without a significant difference $(P>0.10)$; similar findings were observed in normal rats $(r=0.977, P<0.001)$ and phenobarbital-treated rats $(r=0.947, P<0.001)$ (Fig. 6). The data indicate that the displacement of the labeled albumin curve in relation to the labeled RBC curve is compatible with a flow-limited kind of behavior in cirrhotic as well in noncirrhotic rats, as previously reported in cirrhotic patients (6).

Group $A$. In these seven rats, labeled sucrose and water curves were unimodal (Fig. 2). As illustrated in Fig. 7, there was a significant correlation between the extravascular volume for sucrose, calculated using the peak-time concentration, and that calculated using transit time $(r=0.976, n=7, P<0.001)$ without a significant difference $(P>0.10)$; similar findings were observed in normal rats $(r=0.970, P<0.001)$ and phenobarbital-treated rats $(r=0.950, P<0.001)$ (Fig. 7$)$. There was a significant correlation between the values calculated for the extravascular volume of distribution for water $(r=0.986, n=6, P<0.001)$. However, values using the peak-time concentration were slightly higher than those using transit time in cirrhotic; similar findings were observed in normal rats $(r=0.935, n=7, P<0.01)$ and phenobarbital-treated rats $(r=0.805, n=6, P<0.05)$ (Fig. 7).

In these seven cirrhotic rats, data indicated that sucrose and water were still able to diffuse into an extravascular space not accessible to albumin and that diffusion was still flow limited, without apparent restricted permeability.

Group B. In these rats, labeled sucrose curves were bimodal, while water curves remained unimodal (Fig. 3). In each case, the labeled sucrose upslope was almost superimposed on that of labeled RBC and albumin, the peak was slightly lower and slightly ahead of that of the labeled albumin in time, and the down-slope exhibited a biexponential characteristic. The apparent extravascular volume accessible to sucrose calculated using the peak-time concentration differed systematically from the one calculated using the transit time (Fig. 7), which indicates that the sucrose distribution was no longer flow limited in these rats. The distribution of labeled water still appears to be flow limited, as illustrated in Fig. 7.

Group $C$. In these five cirrhotic rats, both labeled sucrose and water curves were bimodal, their peak concentrations being slightly ahead of that of the labeled albumin in time (Fig. 4). The apparent transit time volumes for both sucrose and water distribution were systematically different from the apparent peaktime volume (Fig. 7), which indicates that the distribution of both labels was not flow limited. 


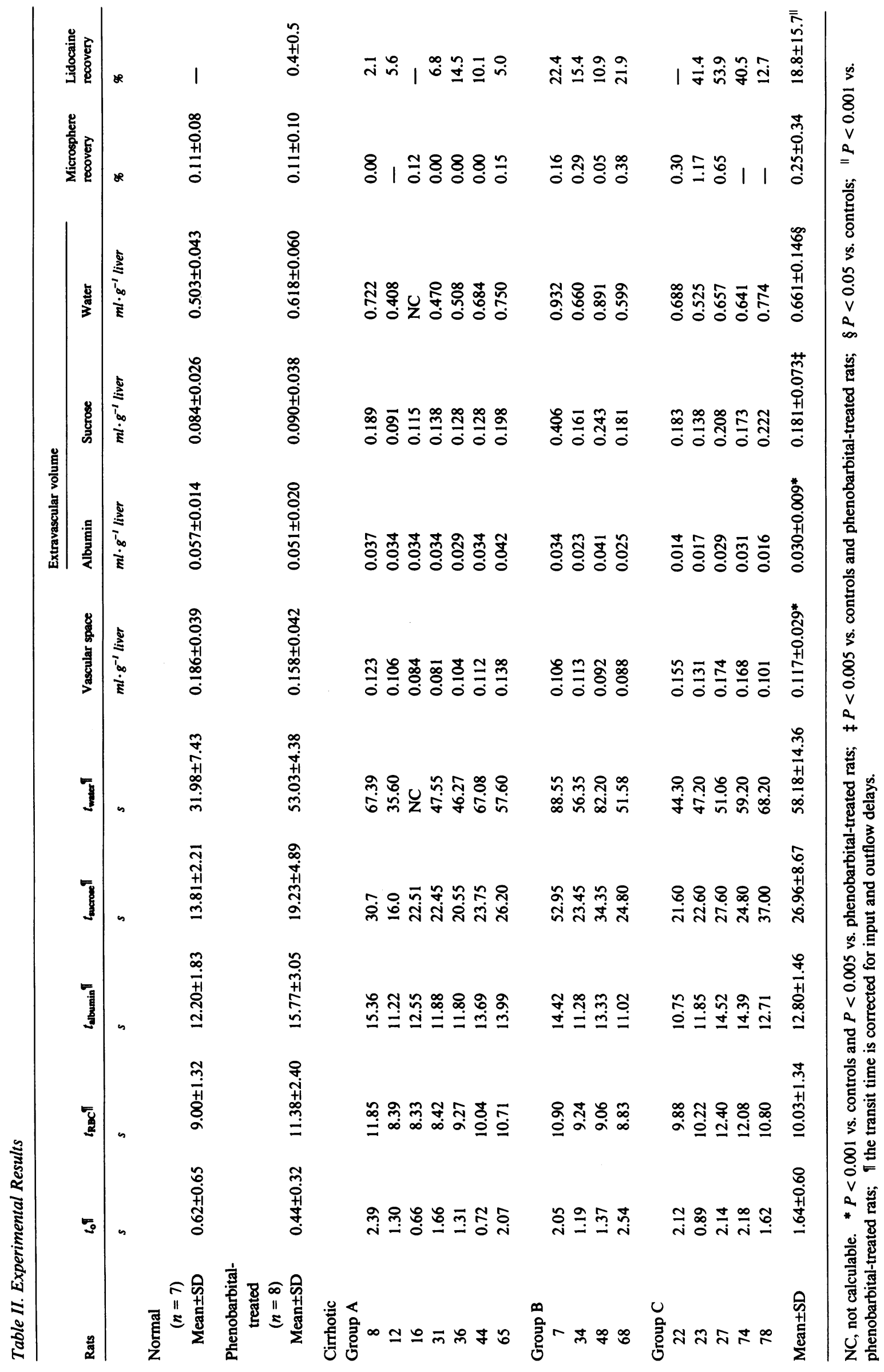




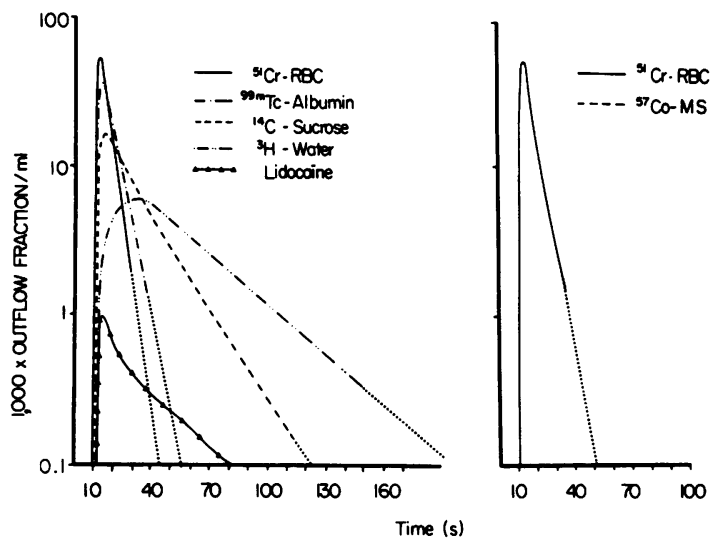

Figure 2. Multiple indicator dilution pattern (left) and outflow recovery of microspheres (MS) (right) obtained in an in situ perfused cirrhotic rat liver from group A (no. 31). The outflow recovery of lidocaine was $6.8 \%$; that of microspheres was $0 \%$.

When considering all cirrhotic rats together, the extravascular volume accessible to sucrose was significantly increased when compared with normal and phenobarbital-treated rats $(P$ $<0.005)$. The extravascular volume accessible to water was slightly increased compared with normal $(P<0.05)$ and phenobarbital-treated rats $(P>0.10)$. However, the total volume accessible to labeled sucrose and water was not significantly different from values obtained in noncirrhotic rats $(P>0.10)$ (Fig. 5).

Microsphere studies. Labeled $15-\mu \mathrm{m}$ microsphere studies were carried out in 13 of the cirrhotic rats; the hepatic venous outflow recovery was $0.25 \pm 0.34 \%$ and the calculated value was $>1.0 \%$ in only one case (rat 23, group C, Fig. 4). There was a slight increase in microsphere recovery from group $\mathrm{A}$ to group $C$, but there was no difference compared with noncirrhotic rats $(P>0.10)$ (Table II). As shown in Figs. 3 and 4, the microsphere outflow pattern never attained the profile of a dilution curve.

Lidocaine studies. Lidocaine studies were performed in 14 of the cirrhotic rats (Table II). In all cases, the unchanged lidocaine outflow pattern had the form of a dilution curve: the

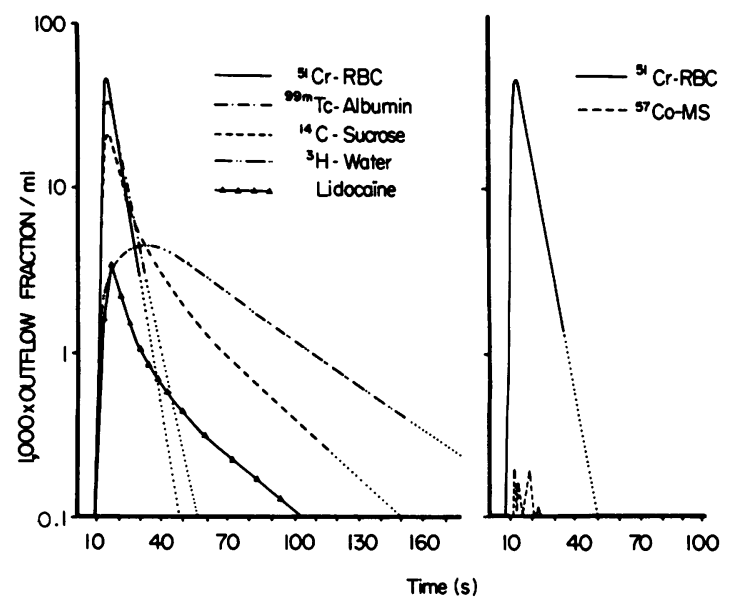

Figure 3. Multiple indicator dilution pattern (left) and outflow of microspheres (MS) (right) obtained in an in situ perfused cirrhotic rat liver from group B (no. 34). The outflow recovery of lidocaine was $15.4 \%$; that of microspheres was $0.29 \%$.
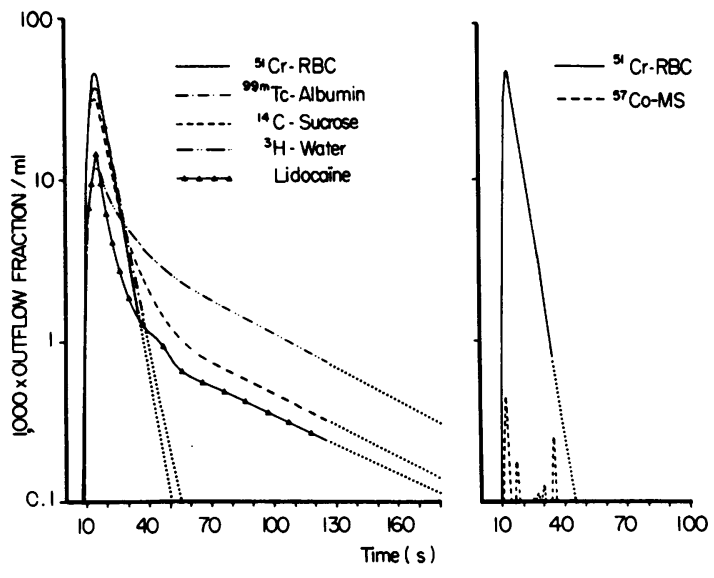

Figure 4. Multiple indicator dilution pattern (left) and outflow recovery of microspheres (MS) (right) obtained in an in situ perfused cirrhotic rat liver from group $C$ (no. 23). The outflow recovery of lidocaine was $41.4 \%$; that of microspheres was $1.17 \%$.

up-slope was slightly delayed in relation to that of labeled RBC curve, the peak concentration was lower and appeared slightly earlier than that of labeled albumin, and the down-slope exhibited a biexponential characteristic. This pattern differed systematically in all cirrhotic rats from that observed in noncirrhotic rats, in which unchanged lidocaine emerged substantially later than labeled water, and was found only in trace amounts. When considering all cirrhotic rats together, lidocaine recovery was markedly increased compared with noncirrhotic rats $(P<0.001)$. Moreover, lidocaine recovery progressed from $7.35 \%$ in group A, to $17.65 \%$ in group B and $37.13 \%$ in group C.

Morphological findings in the cirrhotic liver. By light microscopy, features of cirrhosis were evident in all $\mathrm{CCl}_{4}$-treated rats, with diffuse fibrous septa completely circumscribing nodules of hepatocytes. The ultrastructural examination of cirrhotic nodules showed that most of the sinusoids remained normal with a widened space of Disse containing abundant collagen

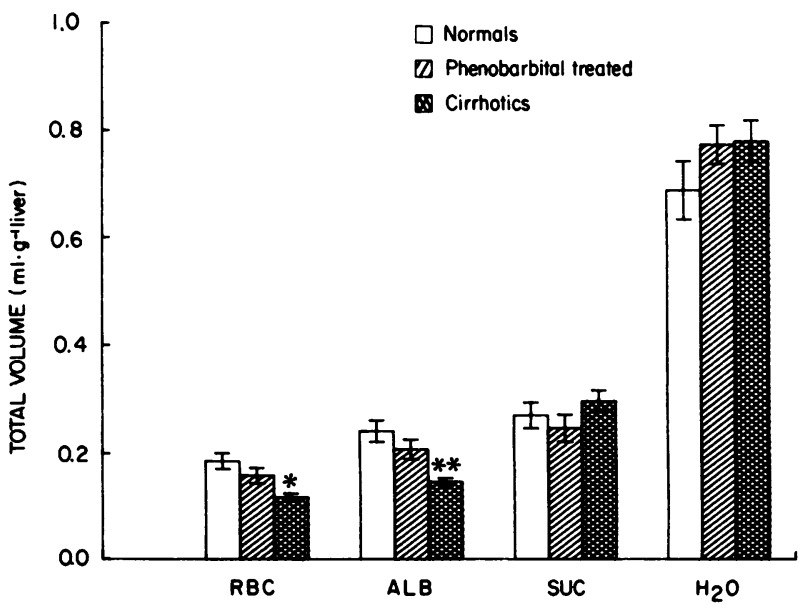

Figure 5. Histogram representing the total volume of distribution for each indicator in normal $(n=7)$, phenobarbital-treated $(n=8)$, and cirrhotic $(n=16)$ rats (mean \pm SEM). ${ }^{*} P<0.001$ when compared with normal and $P<0.005$ when compared with phenobarbital-treated rats; ${ }^{* *} P<0.001$ when compared with normal and phenobarbitaltreated rats. 


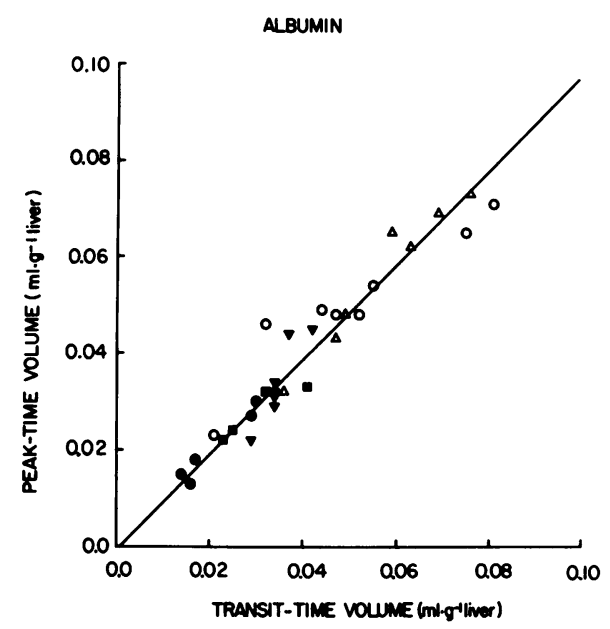

Figure 6. Plots of the extravascular volume of distribution of labeled albumin calculated using the peak-time concentration (abscissa) and the mean transit time (ordinate). The solid line is the straight-line regression through the data obtained in cirrhotic rats; $\Delta$, group A; $\mathbf{n}$, group B; $\bullet$, group $C$. The correlation coefficient for the data is 0.916 $(n=16, P<0.001)$. Values obtained in the seven normal rats $(\Delta)$ and the eight phenobarbital-treated rats $(0)$ are also shown.

fibers. Near the marginal regenerative area, occasional sinusoids took on the appearance of capillaries with continuous endothelial lining lacking fenestrae, supported by a basement membrane and collagen tissue. In some specimens, new capillarized vessels were found in thin fibrotic bands interposed between parenchymal cell cords.

\section{Discussion}

In normal liver, all afferent blood drained by the portal vein has to flow through the sinusoids before reaching the hepatic veins. Within the sinusoids, RBCs are larger than the fenestrae perforating the endothelial cells (20-200 nm diam) (14) and do not have access to the space of Disse, which is freely accessible to molecules contained in plasma. Goresky's (5) approach assumes

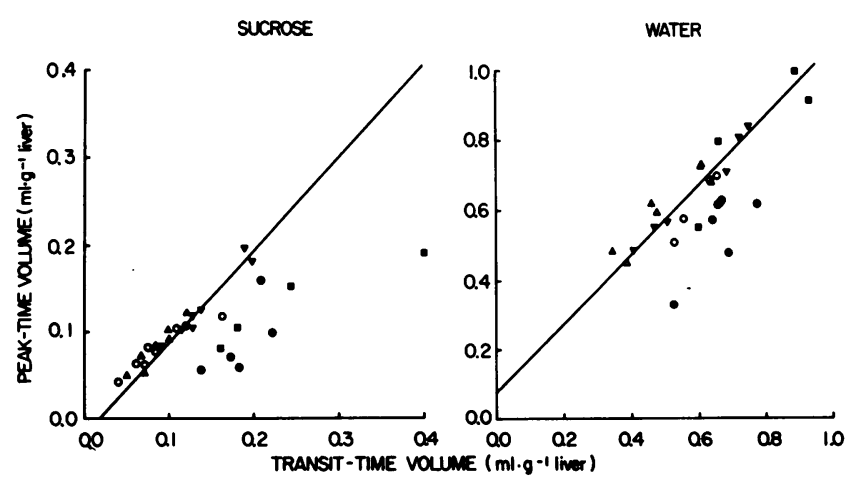

Figure 7. Plots of the extravascular volumes of distribution of labeled sucrose and water calculated using the peak-time concentration (abscissa) and the mean transit time (ordinate). The solid line is the straight-line regression through the data obtained in cirrhotic rats from group A ( $\triangle)$. The correlation coefficient is $0.976(n=7, P<0.001)$ for sucrose data and $0.986(N=6, P<0.001)$ for water data. Values obtained in normal $(\Delta)$, phenobarbital-treated $(0)$, and cirrhotic rats from group B (ø) and group $C(\bullet)$ are also shown. that the distribution of plasma-dissolved substances in the extravascular space is flow limited, and consequently, the volume of their accessible space can be estimated from the displacement of their outflow curves in relation to that of RBCs. This approach depends on the symmetry in the time-concentration changes of the diffusible label curve shape when compared with that of the RBC curve. Indeed, we found that the diffusion of labeled albumin, sucrose, and water (substances not metabolized by the liver) was compatible with flow-limited distribution in normal as well as phenobarbital-treated rats. Our values for the sinusoidal vascular space $\left(V_{\sin }\right)$ and for albumin space are similar to those reported by others in the in situ liver perfusion $(15,16)$; sucrose and water distribution volumes are of the same order of magnitude as those reported in normal dog liver $(5,17)$. The vascular and extravascular volumes of the distribution of various labels were similar in phenobarbital-treated and normal rats when data were expressed per gram of liver weight.

In addition, we used lidocaine to assess the barrier at the liver cell surface. Lidocaine is a highly lipophilic substance that has been found to penetrate the liver cell membrane freely and completely during the first passage through the normal dog liver (18); similar behavior has been reported for monohydric alcohols (17). Lidocaine shows a similar pattern in phenobarbital-treated rats, with its hepatic venous outflow curve emerging substantially later than the labeled water curve. Since lidocaine is normally metabolized by the mixed oxidase system, the small fraction recovered in the outflow may be interpreted as unchanged lidocaine released from intrahepatic binding sites before enzymatic processing.

Using the multiple indicator dilution technique, we have explored events occurring in the microvascular bed of 16 rats with $\mathrm{CCl}_{4}$-induced cirrhosis. This model is known to reproduce most of the features of the human disease (7). In all cases, a finely nodular liver was observed, and light microscopy revealed connective tissue septa surrounding nodules of regenerating hepatocytes resembling human micronodular cirrhosis.

In cirrhotic rats, the sinusoidal vascular space $\left(V_{\text {sin }}\right)$ was significantly reduced when compared with noncirrhotic rats (Table II). Using a similar approach in a preliminary study, Reichen and Le (19) observed no such difference; however, their values for the $\mathrm{RBC}$ space in controls $\left(0.081 \mathrm{ml} \cdot \mathrm{g}^{-1}\right.$ liver $)$ appear to be much lower than previously reported values $(15,16)$ or values obtained in our own noncirrhotic rats (Table II).

In the 16 cirrhotic rats, unimodal outflow dilution curves were obtained for labeled albumin and were compatible with a flow-limited distribution of albumin into its distribution space. However, there was a wide range in the extravascular space accessible to albumin, which indicates that in some of the cirrhotic livers, virtually all the labeled albumin remained in the vascular compartment along with the RBCs. There was a significant decrease in the extravascular space, as well as in the total space accessible to labeled albumin, in cirrhotic as compared with noncirrhotic rats (Table II and Fig. 5).

Data obtained from labeled sucrose and water studies showed that the diffusion of sucrose and water, unlike that of albumin, was not always compatible with a flow-limited system. In seven cirrhotic rats, (classified in group A), both labeled sucrose and water dilution curves showed an unimodal pattern. In these seven rats, the diffusion of sucrose and water was still compatible with flow-limited distribution. It appears that sucrose and water, which are much smaller molecules than albumin, were still able to 
diffuse into an extravascular space inaccessible to albumin. In the other cirrhotic rats, labeled sucrose (groups B and C) and water (group C) dilution curves showed a biexponential pattern and their diffusion was no longer compatible with flow-limited distribution: the volume of distribution calculated from peaktime concentrations (peak-time volume) differed systematically from those calculated from the transit times (transit-time volume) (Fig. 7).

In cirrhotic rats, the extravascular volumes accessible to sucrose and water, as measured by the transit-time method, were significantly higher than in noncirrhotic rats (Table II). However, the total spaces accessible to sucrose and water did not differ from those measured in noncirrhotic rats (Fig. 5).

In rats from groups $\mathrm{B}$ and $\mathrm{C}$, the sucrose and water outflow profiles were close to those observed in capillary circulations with poor permeability to sucrose and water, such as the coronary circulation in the dog (20). In this organ, the labeled sucrose and water outflow profiles can be resolved in two parts: a throughput component, interpreted as label that has come through the coronary circulation without leaving the capillaries, and an exchanging component, interpreted as label that has penetrated an extravascular space, emerging later (20). The labeled sucrose and water curves obtained in cirrhotic rats may well have resulted from a similar phenomenon, with diffusion of both labels being limited across capillarized sinusoids. At the same time, the potential contribution of intrahepatic shunts has to be evaluated, since similar patterns may also have resulted from the transit through two different pathways: a first component related to part of the labeled substances flowing through intrahepatic shunts, and a second, or later, component, related to part of the substances coming through a more normal sinusoidal bed.

In our studies, the presence of intrahepatic large vessel shunting was also directly appraised by the simultaneous use of labeled microspheres ( $15 \mu \mathrm{m}$ diam). In no case was the recovery from the outflow of injected labeled microspheres into the portal vein $>1.2 \%$. Moreover, outflows did not attain the profile of dilution curves in any of the rats, cirrhotic or noncirrhotic, and the late appearance of activity might represent breakdown products smaller than the sinusoidal diameter. The existence of large intrahepatic shunts (10-200 $\mu \mathrm{m}$ diam) between afferent and efferent vessels have been well documented in cirrhotic rats using vascular corrosion casts $(2,3)$. However, cast preparation requires high pressure injection in order to obtain satisfactory penetration of all vascular systems. Extrapolation of such findings to the in vivo situation should therefore be viewed with caution.

Nevertheless, in the cirrhotic livers studied, large vessel shunting could not be demonstrated using $15-\mu \mathrm{m}$ microspheres; the possible development of small channels with poorly permeable walls was then appraised using lidocaine. In all cirrhotic rats, the lidocaine outflow pattern differed systematically from that observed in noncirrhotic rats. It appeared that lidocaine was no longer able to penetrate the liver cell membranes freely and completely, and that a substantial proportion emerged in the outflow related in time to the peak RBC curve. The early peak of unchanged lidocaine can be interpreted as part of the substance flowing through poorly permeable channels, where there are no hepatocytes without leaving the vascular bed. The proportion of lidocaine recovered in the outflow varied greatly in cirrhotic rats and appeared to increase with the progression of liver disease.
As discussed above, the throughput lidocaine might also have resulted from capillarization of sinusoids changing the liver microcirculation in a capillary system. Similar lidocaine patterns were found following one passage through the dog brain, an organ with a capillary circulation (Huet, P.-M., unpublished data). However, such a stereotyped alteration of hepatic sinusoids should have been easily demonstrated by light and electron microscopic studies. In the biopsy specimens examined from all cirrhotic rat livers, we found vascular changes similar to those previously reported in this model $(21,22)$ and, in particular, $(a)$ most of the hepatic sinusoids remained structurally unaltered, the space of Disse being widened with extensive collagen disposition, and $(b)$ occasional capillarized channels were found in some thin fibrotic bands dissecting the parenchymal nodules as well in the internodular fibrotic septa. These vascular channels were similar in diameter to more normal sinusoids. Quantitative morphometric analysis is necessary to further define these alterations and therefore no attempt was made to correlate the morphologic findings and dilution data in the present study. However, this task might be difficult since electron microscopic grids are very small and might not be representative of the entire liver.

Our data are not at variance with the ultrastructural morphometric analysis of rat livers with $\mathrm{CCl}_{4}$-induced cirrhosis published recently (22), in which liver hypertrophy was attributed mainly to an increase in connective tissue with an increased quantity of blood vessels in the septa. In the present study, the increased $t_{\mathrm{o}}$ measured in cirrhotic rats (Table II) also indicates an increase in large vessel volume when compared with noncirrhotic rats. The sinusoidal space was also found to be significantly decreased (22).

Such ultrastructural alterations found in $\mathrm{CCl}_{4}$-induced cirrhosis differ from those reported in human cirrhosis, in which capillarization of sinusoids with the development of a basement membrane is a prominent feature, particularly at the periphery of nodules (23-25). These ultrastructural differences might explain the different labeled sucrose outflow patterns observed in $\mathrm{CCl}_{4}$-induced cirrhotic rats and cirrhotic patients, where the diffusion of labeled sucrose in the extravascular space appeared to be barrier limited (6).

Using the multiple indicator dilution technique, we defined events occurring in the vascular bed of rats with $\mathrm{CCl}_{4}$-induced cirrhosis and found major alterations in liver microcirculation. The data are compatible with two kinds of alteration: $(a)$ a decreased vascular space with collagenization of the extravascular space limiting the diffusion of large molecules such as albumin; and $b$ ) the development of small channels with poorly permeable walls limiting the diffusion of small molecules such as lidocaine, sucrose, and water. Large intrahepatic shunts are not a common feature in this animal model. The combination of these two kinds of alteration may account for the progressive limitation of exchange in the microvascular bed found in cirrhotic rat liver (26).

\section{Acknowledgments}

The authors thank Antoine Brault for his superb technical assistance in liver perfusion, Robert Boileau and François Carrière for their assistance in data analysis, and Manon Bourcier for her secretarial assistance. Light and electron microscopy specimens were handled by Jocelyne Belgiorno.

The financial support for these studies was provided by the Medical Research Council of Canada and the Canadian Liver Foundation. 


\section{References}

1. Schaffner, F., and H. Popper. 1963. Capillarization of hepatic sinusoids. Gastroenterology. 44:239-242.

2. Popper, H., H. Elias, and D. E. Petty. 1952. Vascular pattern of the cirrhotic liver. Am. J. Clin. Pathol. 33:717-729.

3. Mitra, S. K. 1966. Hepatic vascular changes in human and experimental cirrhosis. J. Pathol. Bacteriol. 92:405-415.

4. Popper, H., and W. Orr. 1970. Current concepts in cirrhosis. Scand. J. Gastroenterol. 5(Suppl. 6):203-222.

5. Goresky, C. A. 1963. A linear method for determining liver sinusoidal and extravascular volume. Am. J. Physiol. 204:626-640.

6. Huet, P. M., C. A. Goresky, J. P. Villeneuve, D. Marleau, and J. O. Lough. 1982. Assessment of liver microcirculation in human cirrhosis. J. Clin. Invest. 70:1235-1244.

7. Proctor, E., and K. Chatamra. 1982. High yield micronodular cirrhosis in rat. Gastroenterology. 83:1183-1190.

8. Chinard, F. P., T. Enns, and M. F. Nolan. 1962. Pulmonary extravascular water volumes from transit-time and slope data. J. Appl. Physiol. 17:179-183.

9. Wagner, H. N. Jr. 1968. Technical details of common procedures. In Principles of Nuclear Medicine. H. N. Jr. Wagner, editor. W. B. Saunders, Philadelphia. 832-858.

10. Huet, P. M., and J. P. Villeneuve. 1983. Determinants of drug disposition in patients with cirrhosis. Hepatology. 3:913-918.

11. Herz, R., B. Cueni, J. Bircher, and G. Paumgartner. 1973. The excretory capacity of the isolated rat liver: an in vitro-in vivo comparison. Naunyn-Schmiedeberg's Arch. Pharmacol. 277:297-304.

12. Tavoloni, N., J. S. Reed, and J. L. Boyer. 1978. Hemodynamic effects on determinants of bile secretion in isolated rat liver. Am. J. Physiol. 234(6):E584-E592.

13. Meier, P., and K. L. Zierler. 1954. On the theory of the indicatordilution method for measurement of flow and volume. J. Appl. Physiol. 6:631-744.

14. Wisse, E., R. B. DeZanger, R. Jacobs, and R. S. McCuskey. 1983. Scanning electron microscopic observation on the structure of portal veins, sinusoids and central veins in rat liver. Scanning Electron Microsc. III: $1441-1452$.

15. Wolkoff, A. W., C. A. Goresky, J. Sellin, Z. Gatmaitan, and I. M. Arias. 1979. Role of ligandin in transfer of bilirubin from plasma into liver. Am. J. Phyșiol. 236(6):E638-E648.

16. Reichen, J., and G. Paumgartner. 1976. Uptake of bile acids by perfused rat liver. Am. J. Physiol. 231(3):734-742.

17. Goresky, C. A., E. R. Gordon, and G. G. Bach. 1983. Uptake of monohydric alcohols by liver: demonstration of a shared enzymic space. Am. J. Physiol. 244(7):G198-G214.

18. Huet, P. M., and B. Leclair. 1981. Hepatic handling of lidocaine during a single passage through the liver in dogs. Clin. Invest. Med. 4: 7B. (Abstr.)

19. Reichen, J., and M. Le. 1983. Sinusoidal capillarization is more important than intrahepatic shunting to explain decreased propranolol clearance in liver cirrhotic rats. Gastroenterology. 84:1283. (Abstr.)

20. Rose, C. P., C. A. Goresky, and G. G. Bach. 1977. The capillary and sarcolemnal barrier in the heart. An exploration of labeled water permeability. Circ. Res. 41:515-533.

21. Stenger, R. J. 1966. Hepatic sinusoids in carbon tetra-chloride. Induced cirrhosis. Arch. Pathol. 81:439-447.

22. Ryoo, J. W., and R. J. Buschmann. 1983. A morphometric analysis of the hypertrophy of experimental liver cirrhosis. Virchows Arch. A Pathol. Anat. Histopathol. 400:173-186.

23. Phillips, M. J., and J. W. Steiner. 1965. Electron microscopy of liver cells in cirrhotic nodules. Am. J. Pathol. 46:985-1005.

24. Lapis, K. 1979. Cirrhosis. In Electron Microscopy in Human Medicine, Vol. 8: The Liver, The Gallbladder and Bile Ducts. J. Johannessen, editors. McGraw-Hill Inc., New York. 158-187.

25. Orrego, H., L. M. Blendis, I. R. Grossley, A. Medline, A. MacDonald, S. Ritchie, and Y. Israel. 1981. Correlation of intrahepatic pressure with collagen in the Disse space and hepatomegaly in humans and in the rat. Gastroenterology. 80:546-565.

26. Wood, A. J. J., J. P. Villeneuve, R. A. Branch, L. W. Rogers, and D. G. Shand. 1979. Intact hepatocyte theory of impaired drug metabolism in experimental cirrhosis of the liver. Gastroenterology. 76: $1358-1362$. 